\title{
Repeated Intrathecal Administration of Ropivacaine Hydrochloride Induces Apoptosis Via Up-Regulating of Fas/FasL Expression in the Rat Spinal Cord
}

Fuyu Zhang

Xiangyang No.1 People's Hospital

Lian Zeng

Xiangyang No.1 People's Hospital

Zhao Luo

Xiangyang No.1 People's Hospital

Ming Sang

Hubei Key Laboratory of Wudang Local Chinese Medicine Research

Xiaodong Sun

Hubei Key Laboratory of Wudang Local Chinese Medicine Research

Huiyu Luo ( $\nabla 603983267 @ q q . c o m$ )

Hospital General de Zona No 1

Research article

Keywords: ropivacaine, intrathecal, apoptosis, Fas, FasL

Posted Date: September 25th, 2020

DOI: https://doi.org/10.21203/rs.3.rs-64808/v1

License: (9) (i) This work is licensed under a Creative Commons Attribution 4.0 International License.

Read Full License 


\section{Abstract}

Background: Ropivacaine hydrochloride $(\mathrm{RH})$ is a local anesthetic and frequently used for perioperative anesthesia and analgesia; but it has potential neurotoxicity, especially when intrathecally used repeatedly for a long time, although the exact mechanism is unclear.

Methods: In this study, the expression of Factor associated suicide receptor (Fas) and its ligand (FasL) in the spinal cord cells was detected in rats receiving repeated intrathecal injection of $\mathrm{RH}$. The paw mechanical withdraw threshold (MWT) was measured before and 3 days after intrathecal cannulation and $24 \mathrm{~h}$ after intrathecal administration. Rats received intrathecal injection of $0.5 \%, 1 \%, 2 \% \mathrm{RH}$ at 0.12 $\mathrm{ml} / \mathrm{kg}$ or saline of equal volume alone. Intrathecal injection was done 8 times with an interval of $1.5 \mathrm{~h}$ in $12 \mathrm{~h}$. The spinal cord was collected for pathological examination; TUNEL staining was used to assess the apoptosis in the spinal cord and the expression of Fas, FasL, caspase-3, and caspase- 8 was detected by qPCR and Western blotting.

Results: $1 \%$ and $2 \%$ RH groups significantly increased the MWT $(P<0.05)$ and more vacuoles or edema was observed in the spinal cord after $\mathrm{RH}$ treatment. The apoptosis rates and expression of Fas, FasL, caspase- 3 , and caspase-8 increased in the RH groups $(P<0.05)$.

Conclusions: Repeated intrathecal administration of RH may cause damage to the spinal cord and induce apoptosis in the spinal cord via up-regulating Fas/FasL expression.

\section{Background}

Ropivacaine hydrochloride (RH), as an amino amide local anesthetic (LA), is often used for perioperative nerve blocks, epidural and spinal anesthesia, and pain management (1). However, some studies have suggested that high dose LAs and long LAs exposure may cause symptoms of nerve injury such as transient neurological syndrome (TNS) and cauda equina syndrome (CES) when they are intrathecally used $(2,3)$. Most damages are transient and often subclinical, but some complications may induce dyskinesia and sensory disorder (4). These complications are closely related to the neurotoxicity of LAs $(5,6)$. After nerve injury, the recovery is a long process, and not effective preventive measures have been developed for the nerve injury secondary to repeated intrathecal use of LAs (7).

Currently, the exact mechanism of LAs' neurotoxicity remains unclear, and multiple signaling pathways have been proposed, of which, apoptosis is a main pathophysiological mechanism $(8,9)$. The death receptor pathway is a classical form of exogenous apoptosis, and factor associated suicide receptor (Fas) and its ligand (FasL) play an important role in this pathway. Fas, also known as APO-1 / CD95, is a member of the tumor necrosis factor (TNF) receptor family and widely expressed in a variety of cells and tissues. Fas mainly functions to induce apoptosis and immune regulation $(10,11)$. Fas binding to FasL can activate the Fas-associated death domain (FADD) and procaspase-8, which subsequently activates caspase-3, ultimately leading to apoptosis. 
Fas and FasL can be expressed in the nervous system to induce apoptosis in the spinal cord injury(12); Robins-Steele et al. found that intrathecal administration of soluble Fas receptors (sFas) could inhibit the neuronal apoptosis after spinal cord injury (13). Our previous study confirmed that ropivacaine induced Fas/FasL expression in rat pheochromocytoma PC12 cells (14). However, it is unclear whether Fas / FasL is involved in the neurotoxicity of LAs to the spinal cord.

In this study, rats were intrathecally administrated with $\mathrm{RH}$ at different concentrations, and the expression of Fas/FasL and caspase related proteins was detected in the spinal cord, aiming to investigate whether the Fas/FasL pathway is involved in the $\mathrm{RH}$-induced neuronal apoptosis in the rat spinal cord.

\section{Methods}

Animals

Twenty-five healthy male Sprague-Dawley (SD) rats, weighing 200-300 g and aged 6-8 weeks, were purchased from the Experimental Animal Center of the Hubei University of Medicine. The experiment was approved by the Animal Ethics Committee of Xiangyang No.1 People's Hospital. Rats were housed in an environment at $22 \pm 2{ }^{\circ} \mathrm{C}$ with the relative humidity of $50-60 \%$ and $12 \mathrm{~h}: 12 \mathrm{~h}$ dark-light cycle, and given ad libitum access to food and water. Animals were allowed to accommodate to the environment for 1 week. All procedures performed in studies involving animals were in accordance with the ethical standards of the institution or practice at the Animal Ethics Committee of the Hubei University of Medicine (2018DW003).

Intrathecal catheterization and drug administration

Rats were intraperitoneally anesthetized with $10 \%$ chloral hydrate. According to published method (15), a PE-10 catheter (inner diameter, $0.12 \mathrm{~mm}$; outer diameter, $0.35 \mathrm{~mm}$; Smith Medical, UK) was inserted through the L4/L5 intervertebral space(depth: $2 \mathrm{~cm}$ ) and fixed at the L1-L2 level. The cannulated rats were observed for 3 days. Five rats developing neurological symptoms after cannulation were excluded from this study.

The remaining twenty cannulated rats were randomly divided into 4 groups ( $\mathrm{n}=5$ per group): Saline group, $0.5 \% \mathrm{RH}$ group, $1 \% \mathrm{RH}$ group, and $2 \% \mathrm{RH}$ group. According to the previously reported (16), rats received intrathecal injection of $0.5 \%, 1 \%, 2 \% \mathrm{RH}$ at $0.12 \mathrm{ml} / \mathrm{kg}$ (Hengrui, China) or saline of equal volume alone through the catheter. Intrathecal injection was done 8 times with an interval of $1.5 \mathrm{~h}$ in $12 \mathrm{~h}$. After the last injection, rats in each group were observed for $24 \mathrm{~h}$.

Behavioral assessment

The mechanical paw withdrawal threshold (MWT) was measured with the Electronic von Frey apparatus (Bioseb, France). In brief rats were placed in a transparent plexiglass box with holes at the bottom to limit the range of motion and animals were allowed to adapt to the environment for $15 \mathrm{~min}$. Then, the von Frey probe was used to directly stimulate the skin of the hind paw of the rat and the pressure increased 
gradually. When the rat had behavioral reactions such as raising, licking and screaming, the threshold was recorded as the MWT. All rats were tested at 3-time points: before cannulation, 3 days after cannulation, and $24 \mathrm{~h}$ after intrathecal administration. Each rat was tested 3 times with an interval of 15 min. The specific process of the experiment is shown in Fig. 1.

HE staining

Animals were euthanized by $\mathrm{CO}_{2}$ inhalation. The spinal cord tissues were collected at $24 \mathrm{~h}$ after the last intrathecal injection. Tissues were embedded in paraffin, then sectioned and stained according to the HE kit instructions (Beyotime, China). The morphology of the spinal cord tissues was observed under a light microscope (Olympus, IX73, Japan), and compared among groups.

TUNEL staining

The apoptosis of spinal cord tissue was detected by TUNEL staining (Roche, Germany) according to the manufacturer's instructions. After deparaffinization, rehydration, and treatment with $3 \% \mathrm{H}_{2} \mathrm{O}_{2}$ for 10 min at room temperature, the sections were incubated with proteinase $\mathrm{K}$ (1:200 in Tris-buffered saline [TBS]) for $30 \mathrm{~min}$ at $37^{\circ} \mathrm{C}$ and then treated with Triton-X100 (0.01\%) for $20 \mathrm{~min}$. The sections were subsequently incubated with TdT and dUTP-digoxigenin in a humidified chamber at $37^{\circ} \mathrm{C}$ for $2 \mathrm{~h}$, followed by three washes in TBS. The sections were subjected to nuclear staining with DAPI and then observed under an inverted fluorescence microscope (Olympus, IX73, Japan). 3 randomly selected fields were selected from the posterior horn in each section. The proportion of TUNEL positive cells was determined at a magnification of $200 x$.

Quality real-time PCR (qPCR)

Total RNA was extracted using TRIZOL reagents (Invitrogen, USA) from the spinal cord tissues of rats. The RNA extraction was done according to a standard protocol and then the RNA concentration was determined by NanoPhotometry (Implen, Germany). Subsequently, $2 \mu \mathrm{g}$ of RNA was reverse-transcribed into cDNA according to manufacturer's instructions (Promega, USA). The qPCR was performed using SYBR Green fluorescent kit, and the reaction was performed in an AB17500 Real-time PCR system (Applied Biosystems, USA) with a cycle program as follows: denaturation for 2 min at $95^{\circ} \mathrm{C}, 40$ cycles of $95^{\circ} \mathrm{C}$ for $15 \mathrm{~s}$, and $60^{\circ} \mathrm{C}$ for $1 \mathrm{~min}$. The relative expression of a specific gene was cdetermined with the $2^{-\triangle \triangle C T}$ method. GAPDH was used as an internal control, and the primer sequences used are shown in Table 1. 
Table 1

Primers used for qPCR

\begin{tabular}{|c|c|c|}
\hline Gene & Primer sequence & Product size (bp) \\
\hline Fas & & 105 \\
\hline Forward & 5'-ATGAGATCGAGCACAACAGC-3' & \\
\hline Reverse & 5'-TTAAAGCTTGACACGCACCA-3' & \\
\hline FasL & & 242 \\
\hline Forward & 5'-TTCCTGCGTGGTTTCTTC-3' & \\
\hline Reverse & 5'-CCATGTCCTTTCTACTTTGTCA-3' & \\
\hline Caspase3 & & 155 \\
\hline Forward & 5'-GAAAGCCGAAACTCTTCATCA-3' & \\
\hline Reverse & 5'-ATAGTAACCGGGTGCGGTAG-3' & \\
\hline Caspase8 & & 186 \\
\hline Forward & 5'-TCCGGTGTTTTATAGTTCCGCT-3' & \\
\hline Reverse & 5'-GGGTAGGAGAGCTGTAACCTGT-3' & \\
\hline GAPDH & & 142 \\
\hline Forward & 5'-GGCTACACTGAGGACCAGGTT-3' & \\
\hline Reverse & 5'-TGCTGTAGCCATATTCATTGTC-3' & \\
\hline
\end{tabular}

Western blotting

The spinal cord tissues were lysed with RIPA lysis buffer (Beyotime, China). Then the protein concentration was determined with the bicinchoninic acid (BCA) protein assay kit (Beyotime, China). $50 \mu \mathrm{g}$ of protein was separated in each sample by $10 \%$ SDS polyacrylamide gel electrophoresis and then transferred onto a nitrocellulose (NC) membranes. After blocking in 5\% milk in TBST for $1 \mathrm{~h}$ at room temperature, the membranes were incubated with primary antibodies against Fas (1:1000), caspase-3 (1:1000), and a-tubulin (1:1000) (Proteintech, China) or FasL (1:1000) (Servicebio, China) overnight at $4{ }^{\circ} \mathrm{C}$. Then the membranes were incubated with horseradish-peroxidase-conjugated secondary antibodies (1:5000) (Cell Signaling, USA). Specific proteins were visualized using a chemiluminescence detection system, and the protein bands were quantified with the Image Lab software. The expression of Fas, FasL, and cleaved caspase 3 was normalized to that of a-tubulin as an internal reference.

Statistical analysis 
All assays were performed at least in triplicate, and the data are presented as mean \pm standard deviation (SD). When compared between groups the data were analysed with the $t$ test, one-way ANOVA followed by post hoc Turkey test by SPSS software (version 25.0, IBM, USA). A value of $P<0.05$ was considered statistically significant.

\section{Results}

The MWT of rats was increased by repeated intrathecal RH

The MWT at 3 days after intrathecal catheterization was similar to that before intrathecal catheterization (Fig. 2A, 2B; $P>0.05$ ), but $1 \%$ and $2 \%$ RH groups had significantly higher MWT as compared to the saline group at $24 \mathrm{~h}$ after intrathecal administration $(P<0.05)$.

Repeated intrathecal $\mathrm{RH}$ affected the morphology of spinal cord

HE staining showed the spinal cord structure in each group was relatively complete. In the saline group, there were no morphological abnormalities. The mild edema of the posterior horn was observed in the spinal cord of $0.5 \% \mathrm{RH}$ group, and significant edema was noted in the $1 \%$ and $2 \% \mathrm{RH}$ groups. In addition, some vacuoles and nuclear membranes dissolution were observed in the spinal cord after repeated intrathecal use of RH (Fig. 3).

$\mathrm{RH}$ induced apoptosis in the spinal cord

The results showed a few apoptotic cells in the saline group. However, a lot of apoptotic cells were observed in the posterior horn of the spinal cord of $\mathrm{RH}$ groups $(0.5 \%, 1 \%$ and $2 \%$ ) (Fig. 4A). Compared with the saline group, the proportion of apoptotic cells in the posterior horn increased with the increase in the $\mathrm{RH}$ concentration (Fig. 4B, 4C; $P<0.05$ ).

RH up-regulated mRNA expression of Fas, FasL, Caspase-8, and Caspase-3 in the spinal cord

As shown in Fig. 5A, 5B, 5C, the mRNA expression of Fas, FasL and caspase-8 increased significantly in RH groups $(0.5 \%, 1 \%, 2 \%)$ compared with saline group $(P<0.05)$. There was no statistical difference between $0.5 \% \mathrm{RH}$ group and saline group in the caspase-3 mRNA expression $(P>0.05)$, but caspase-3 mRNA expression in the $1 \%$ and $2 \%$ RH groups was significantly higher than in the saline group (Fig. 5D; $P<0.05)$.

$\mathrm{RH}$ increased protein expression of Fas, FasL, and cleaved caspase-3 in the spinal cord

Results showed intrathecal administration of $\mathrm{RH}$ at different concentrations $(0.5 \%, 1 \%$ and $2 \%)$ for $12 \mathrm{~h}$ significantly increased the protein expression of Fas, FasL, and cleaved caspase-3 as compared to the saline group (Fig. $6 ; P<0.05$ ).

\section{Discussion}


Nerve injury is a complication of peripheral nerve blocking and spinal anesthesia. There are many factors related to the nerve injury, such as the high concentration LAs and inaccurate puncture for anesthesia. Increasing evidence shows that the neurotoxicity of LAs is an important risk factor to the nerve injury (17, 18). $\mathrm{RH}$ is frequently used in the epidural and spinal anesthesia. Compared with bupivacaine, $\mathrm{RH}$ can provide a good analgesic effect with less motor blockade. However, like other LAs, neurotoxicity of RH has been reported in some kinds of animal and cell experiments, and it is closely related to the nerve injury in some cases. However, the exact mechanism of RH neurotoxicity after intrathecal use is currently unclear, and no effective measures have been developed to prevent the nerve injury caused by RH.

In the present study, rats received repeated intrathecal administration of $\mathrm{RH}$ at different concentrations for $12 \mathrm{~h}$, and the apoptosis in the spinal cord was assessed at $24 \mathrm{~h}$ after $\mathrm{RH}$ exposure. Our results showed rats treated with $1 \%$ or $2 \% \mathrm{RH}$ developed symptoms of nerve injury, manifested by an increased MWT. Morphologically, edema and vacuolization increased in the posterior horn of the spinal cord after $\mathrm{RH}$ treatment, and the apoptosis rate of the spinal cord elevated with the increase in the $\mathrm{RH}$ concentration. This suggests that $\mathrm{RH}$ at a high concentration is more likely to induce apoptosis in the spinal cord and symptoms of nerve injury such as paresthesia, which is consistent with the relationship between LA concentration and incidence of nerve injury in the clinical practice (19). Moreover, the expression of Fas and FasL was up-regulated after $\mathrm{RH}$ administration, and the expression of caspase-3 and caspase-8 increased. It indicates that the Fas / FasL pathway is involved in RH induced neuronal apoptosis in the rat spinal cord, which is related to the neurotoxicity of $\mathrm{RH}$.

Apoptosis has been proposed as an important mechanism of LA's neurotoxicity (20). Recent studies have shown that LAs can induce apoptosis through a variety of pathways. In addition to the Fas/FasL pathway, some investigators have reported that $\mathrm{RH}$ can reduce the mitochondrial membrane potential of human neuroblastoma $\mathrm{SH}-5 \mathrm{Y} 5 \mathrm{Y}$ cells, which decreases the cytochrome $\mathrm{C}$ activity and ATP production, thereby activating caspase-3 to induce apoptosis (21). Niu et al. revealed that RH reduced the expression of major mitochondrial regulator peroxisome proliferator-activated receptor gamma coactivator 1-alpha (PGC-1a) and its downstream transcription factors, then inducing the loss of mitochondrial function (22). Zheng et al. found RH induced poly-ADP ribose polymerases-1 (PARP-1) activation and nicotinamide adenine dinucleotide (NAD+) depletion in the SH-SY5Y cells, and induced PARP-1-dependent cell death via apoptosis (23). The study of Wang et al. showed that the miR-210 expression increased after bupivacaine treatment, and miR-210 could target the 3'UTR region of the neurotrophic factor brain-derived neurotrophic factor (BDNF), eventually inducing apoptosis (24). In addition to inducing apoptosis, LAs can also induce neurotoxicity by interfering with autophagy (25) and neuroinflammation (26).

Available studies have demonstrated the role of Fas/FasL in the apoptosis following spinal cord injury, and our study also showed Fas/FasL was involved in the neurotoxicity of RH. The expression of Fas/FasL was regulated via multiple signaling pathways $(27,28)$, and the specific mechanism by which LAs up-regulate Fas/FasL expression is still poorly understood. Sujan et al. revealed that the p38MAPKp53-Fas-Caspases pathway played an important role in the regulation of anti-neoplastic activity of S-allyl cysteine (SAC) (29). Wang et al. found regulating p38 MAPK expression by siRNA technology 
significantly reduced the Fas expression in the neurons such as rat pheochromocytoma PC12 cells and human glioma U87 cells (30). These results suggest that p38 MAPK/p53 may be an important molecular basis for the up-regulated Fas expression. Whether the p38MAPK-p53 signaling pathway is involved in the up-regulation of Fas/FasL expression in the rat spinal cord after $\mathrm{RH}$ is warranted to be further studied.

\section{Conclusions}

In summary, our study suggests that repeated intrathecal RH administration can induce symptoms of neurological damage, which may be related to the apoptosis in rat spinal cord via an Fas/FasL dependent manner. Our findings provide an experimental basis for the prevention and treatment of complications of LA neurotoxicity.

\section{List Of Abbreviations}

Ropivacaine hydrochloride (RH); local anesthetic (LA); transient neurological syndrome (TNS); cauda equina syndrome (CES); factor associated suicide receptor (Fas); factor associated suicide receptor ligand (FasL); tumor necrosis factor (TNF); Fas-associated death domain (FADD); soluble Fas receptors (sFas); mechanical paw withdrawal threshold (MWT); oly-ADP ribose polymerases-1 (PARP-1)

\section{Declarations}

\section{Ethics approval and consent to participate}

The experiment was approved by the Animal Ethics Committee of Xiangyang No.1 People's Hospital.

\section{Consent for publication}

Not applicable

\section{Availability of data and materials}

The datasets used and/or analysed during the current study are available from the corresponding author on reasonable request.

\section{Competing interests}

The authors declare that they have no competing interests.

\section{Funding}

This work was supported by the Health and Family Planning Commission of Hubei Province (No. WJ2019F74), and Innovative Research Program for Graduates of Hubei University of Medicine (No. YC2019026). The funding played no role in the design of the study, or collection, analysis, and interpretation of data, or in writing the manuscript. 


\section{Authors' contributions}

FZ designed the work, acquired and analyzed data, and participated in writing the manuscript; LZ, ZL, MS and XS acquired and analyzed data and revised the manuscript; and HL contributed to the concept and design of the work, reviewed and revised the manuscript.

\section{Acknowledgements}

Not applicable

\section{References}

1. Crumley S, Schraag S. The role of local anaesthetic techniques in ERAS protocols for thoracic surgery. J Thorac Dis. 2018:1998-2004.

2. Tseng WC, Wu ZF, Liaw WJ, Hwa SY, Hung NK. A patient with postpolio syndrome developed cauda equina syndrome after neuraxial anesthesia: A case report. J Clin Anesth. 2017;37:49-51.

3. Chen X, Xu Z, Lin R, Liu Z. Persistent cauda equina syndrome after cesarean section under combined spinal-epidural anesthesia: a case report. J Clin Anesth. 2015;27:520-3.

4. Konig A, Amelung L, Danne M, Meier U, Lemcke J. Do we know the outcome predictors for cauda equine syndrome (CES)? A retrospective, single-center analysis of 60 patients with CES with a suggestion for a new score to measure severity of symptoms. Eur Spine J. 2017;26:2565-72.

5. Verlinde M, Hollmann M, Stevens M, Hermanns H, Werdehausen R, Lirk P. Local Anesthetic-Induced Neurotoxicity. Int J Mol Sci. 2016;17:339.

6. Sekimoto K, Tobe M, Saito S. Local anesthetic toxicity: acute and chronic management. Acute Medicine Surgery. 2017;4:152-60.

7. Long B, Koyfman A, Gottlieb M. Evaluation and management of cauda equina syndrome in the emergency department. Am J Emerg Med. 2019:158402.

8. Fan Y-L, Li H-C, Zhao W, Peng H-H, Huang F, Jiang W-H, et al. Curcumin Attenuated BupivacaineInduced Neurotoxicity in SH-SY5Y Cells Via Activation of the Akt Signaling Pathway. Neurochem Res. 2016;41:2425-32.

9. Zhao W, Liu Z, Yu X, Lai L, Li H, Liu Z, et al. iTRAQ proteomics analysis reveals that PI3K is highly associated with bupivacaine-induced neurotoxicity pathways. PROTEOMICS. 2016;16:564-75.

10. Yamada A, Arakaki R, Saito M, Kudo Y, Ishimaru N. Dual Role of Fas/FasL-Mediated Signal in Peripheral Immune Tolerance. Frontiers in Immunology. 2017;8.

11. Brint $E, O$ Callaghan $G$, Houston A. Life in the Fas lane: differential outcomes of Fas signaling. Cell Mol Life Sci. 2013;70:4085-99.

12. Sobrido-Camean D, Barreiro-Iglesias A. Role of Caspase-8 and Fas in Cell Death After Spinal Cord Injury. Front Mol Neurosci. 2018;11:101. 
13. Robins-Steele S, Nguyen DH, Fehlings MG. The Delayed Post-Injury Administration of Soluble Fas Receptor Attenuates Post-Traumatic Neural Degeneration and Enhances Functional Recovery after Traumatic Cervical Spinal Cord Injury. J Neurotrauma. 2012;29:1586-99.

14. Luo Z, Zhang Z, Zhang F, Liu Y, Zhang Y, Sun X, et al. Ropivacaine mesylate exerts neurotoxicity via up-regulation of Fas/FasL expression in rat pheochromocytoma PC12 cells. American journal of translational research. 2019:1626-34.

15. Hou Y, Wang L, Gao J, Jin X, Ji F, Yang J. A modified procedure for lumbar intrathecal catheterization in rats. Neurol Res. 2016;38:725-32.

16. SUN ZH. Repeated intrathecal administration of ropivacaine causes neurotoxicity in rats. Anaesthesia and Intensive Care \%\2018-05-07 16:32:00. 2012.

17. Perez-Castro R, Patel S, Garavito-Aguilar ZV, Rosenberg A, Recio-Pinto E, Zhang J, et al. Cytotoxicity of Local Anesthetics in Human Neuronal Cells. Anesthesia Analgesia. 2009;108:997-1007.

18. Yu X-j, Zhao W, Li Y-j, Li F-x, Liu Z-j, Xu H-l, et al. Neurotoxicity Comparison of Two Types of Local Anaesthetics: Amide-Bupivacaine versus Ester-Procaine. Sci Rep. 2017;7:45316.

19. Sondekoppam RV, Tsui BCH. Factors Associated With Risk of Neurologic Complications After Peripheral Nerve Blocks. Anesthesia Analgesia. 2017;124:645-60.

20. Wen X, Liang H, Li H, Ou W, Wang H-B, Liu H, et al. In vitro neurotoxicity by ropivacaine is reduced by silencing Cav3.3 T-type calcium subunits in neonatal rat sensory neurons. Artificial Cells, Nanomedicine, and Biotechnology. 2017:1-8.

21. Chen $Y$, Yan $L$, Zhang $Y$, Yang $X$. The role of DRP1 in ropivacaine-induced mitochondrial dysfunction and neurotoxicity. Artificial Cells Nanomedicine Biotechnology. 2019;47:1788-96.

22. Niu Z, Tang J, Ren Y, Feng W. Ropivacaine impairs mitochondrial biogenesis by reducing PGC-1a. Biochem Biophys Res Commun. 2018;504:513-8.

23. Zheng T, Zheng C-y, Zheng X-c, Zhao R-g, Chen Y-q. Effect of parthanatos on ropivacaine-induced damage in SH-SY5Y cells. Clin Exp Pharmacol Physiol. 2017;44:586-94.

24. Wang $Y$, Ni H, Zhang W, Wang $X$, Zhang $H$. Downregulation of miR-210 protected bupivacaineinduced neurotoxicity in dorsal root ganglion. Exp Brain Res. 2016;234:1057-65.

25. Xiong J, Kong Q, Dai L, Ma H, Cao X, Liu L, et al. Autophagy activated by tuberin/mTOR/p70S6K suppression is a protective mechanism against local anaesthetics neurotoxicity. J Cell Mol Med. 2017;21:579-87.

26. Periyasamy P, Guo ML, Buch S. Cocaine induces astrocytosis through ER stress-mediated activation of autophagy. Autophagy. 2016;12:1310-29.

27. Wilson AM, Morquette B, Abdouh M, Unsain N, Barker PA, Feinstein E, et al. ASPP1/2 Regulate p53Dependent Death of Retinal Ganglion Cells through PUMA and Fas/CD95 Activation In Vivo. J Neurosci. 2013;33:2205-16.

28. Liu F, Bardhan K, Yang D, Thangaraju M, Ganapathy V, Waller JL, et al. NF-KB Directly Regulates Fas Transcription to Modulate Fas-mediated Apoptosis and Tumor Suppression. J Biol Chem. 
2012;287:25530-40.

29. Chatterjee S, Patra D, Chakraborti U, Sengupta D, Ghosh P, Basu A, et al. Association of p38MAPKp53-Fas aggregation in S-allyl cysteine mediated regulation of hepatocarcinoma. Environ Toxicol. 2019;34:928-40.

30. Wang S, Lin Q, Wang Z, Pan X. Ropivacaine induces neurotoxicity by activating MAPK/p38 signal to upregulate Fas expression in neurogliocyte. Neurosci Lett. 2019;706:7-11.

\section{Figures}

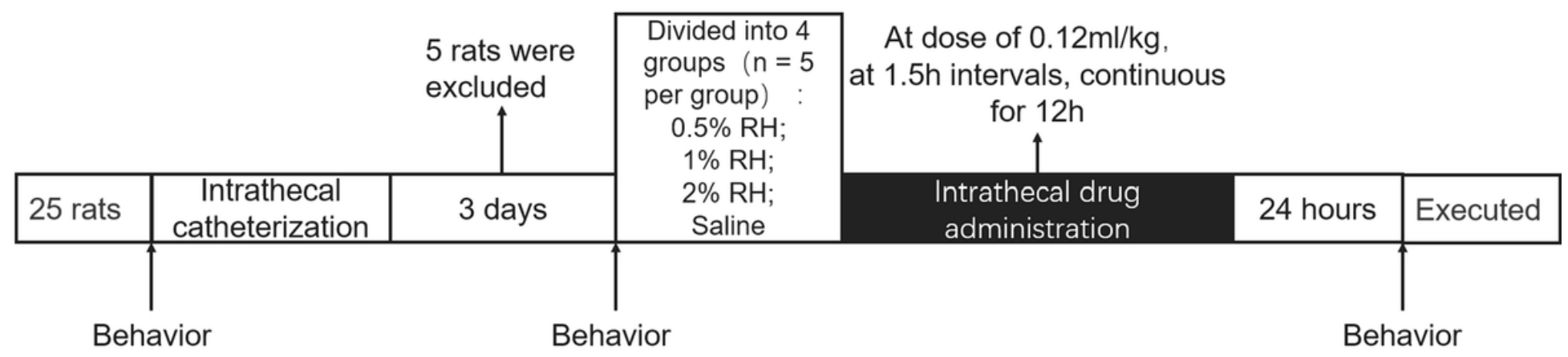

Figure 1

Experimental groups and protocol. Schematic diagram of the four groups of rats exposed to different treatments.
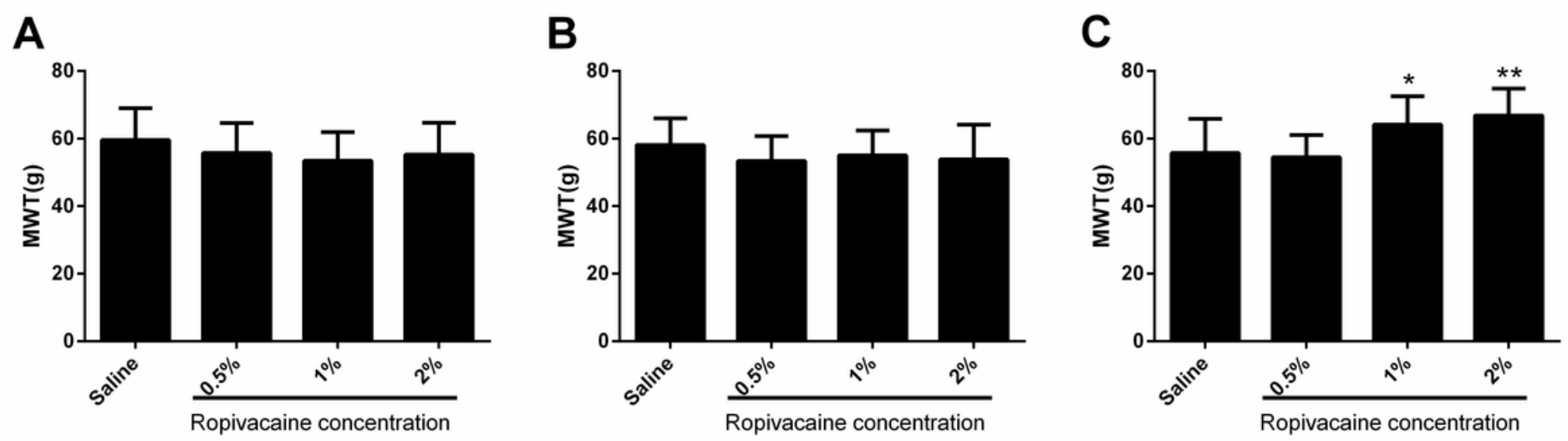

Figure 2

Mechanical paw withdrawal threshold (MWT) of rats in groups at different time points. A. MWT was measured before intrathecal catheterization. B. MWT was measured at 3 days after intrathecal catheterization. C. MWT was measured at $24 \mathrm{~h}$ after intrathecal administration. * $\mathrm{P}<0.05, * * P<0.01$ vs saline group. 

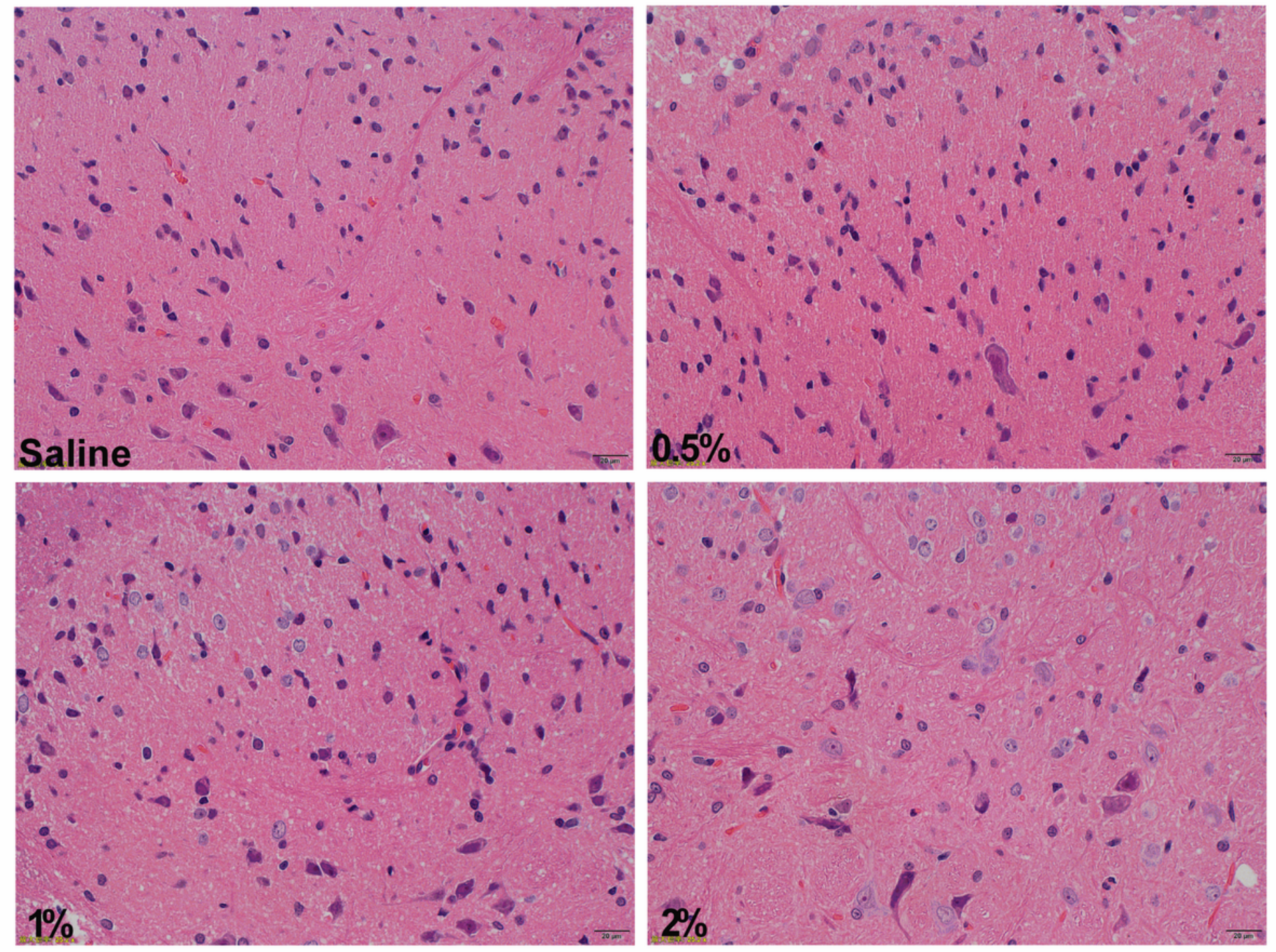

\section{Figure 3}

Pathological changes in the spinal cord after intrathecal administration of $\mathrm{RH}$. Vacuoles and interstitial edema were found in the spinal cord of rats treated with $\mathrm{RH}(40 \times$, scale $=200 \mu \mathrm{m})$. 
A
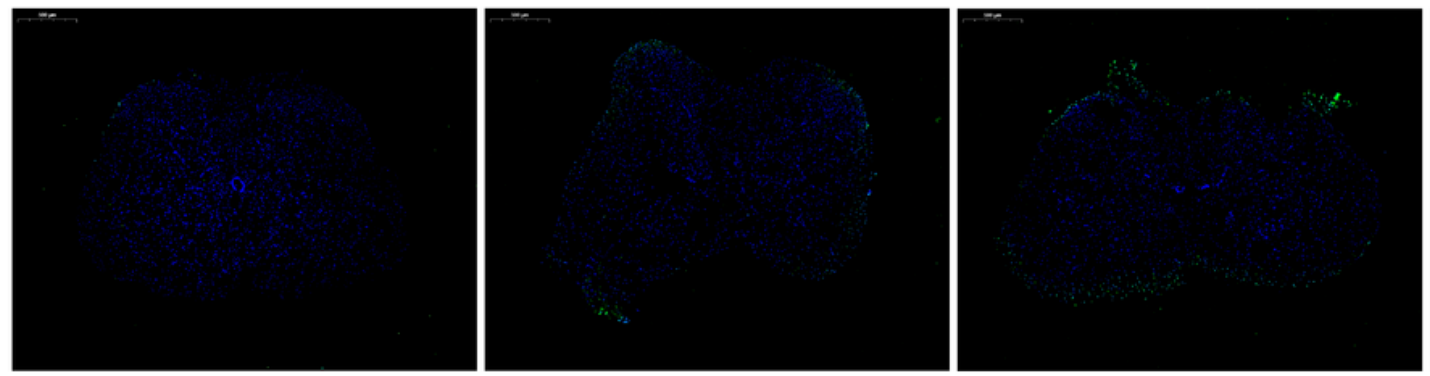

B

Saline

$0.5 \%$

$1 \%$

C
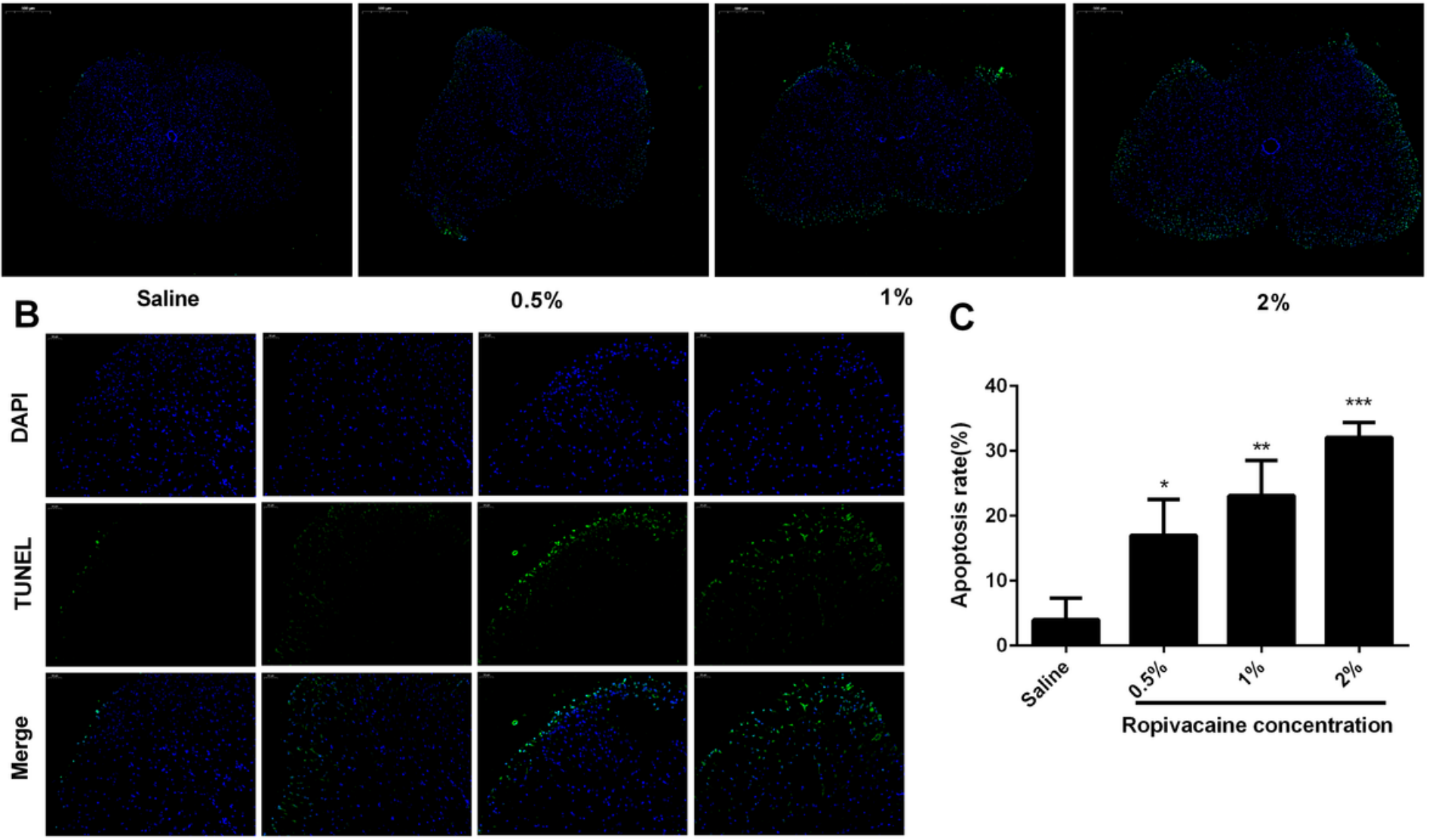

Saline

$0.5 \%$

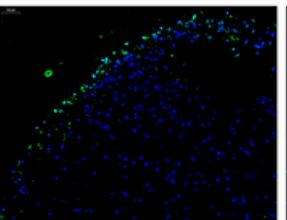

$1 \%$

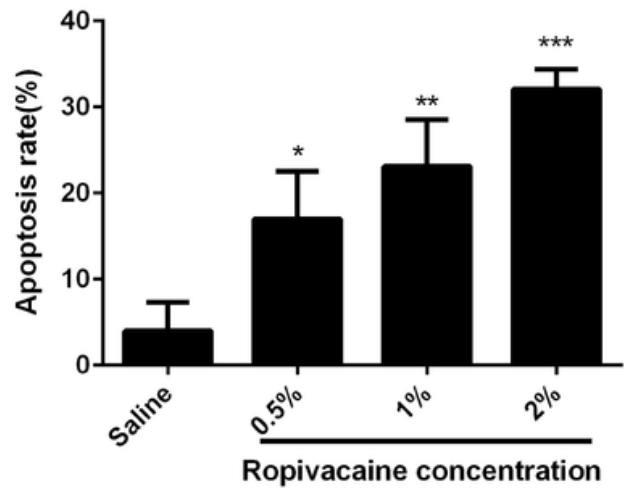

Ropivacaine concentration

\section{Figure 4}

Apoptosis in the spinal cord. TUNEL staining of the spinal cord in each group, A. Morphology at a low magnification $(40 x$, scale $=500 \mu \mathrm{m})$. B. Morphology at a high magnification $(200 x$, scale $=50 \mu \mathrm{m})$; DAPI: nucleus, TUNEL: apoptosis cells. C. Apoptosis rate of each group (mean $\pm S D$ ). ${ }^{*} P<0.05,{ }^{*} P<0.01$, $\star \star \star P<0.001$ vs saline group. 
A
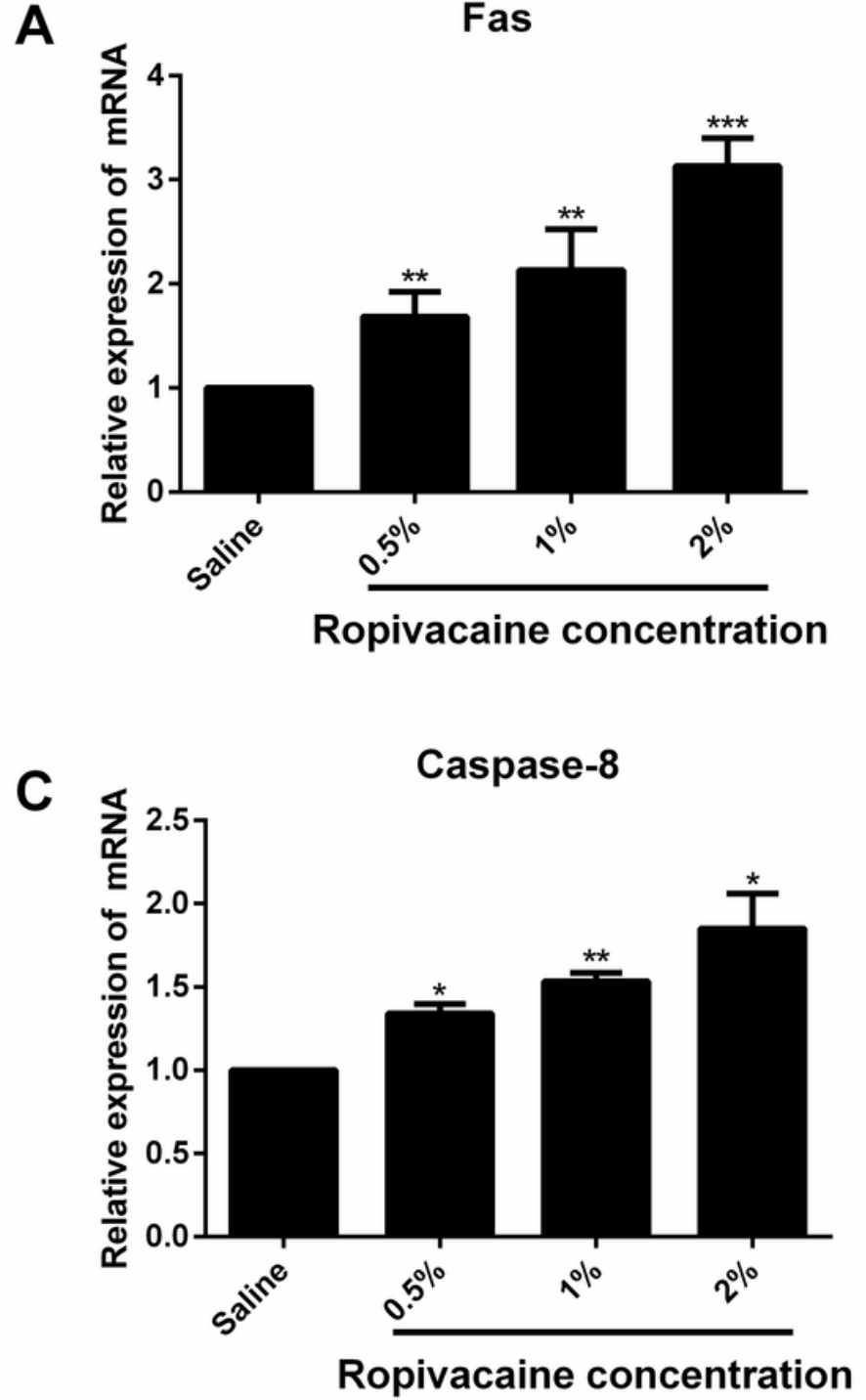

B

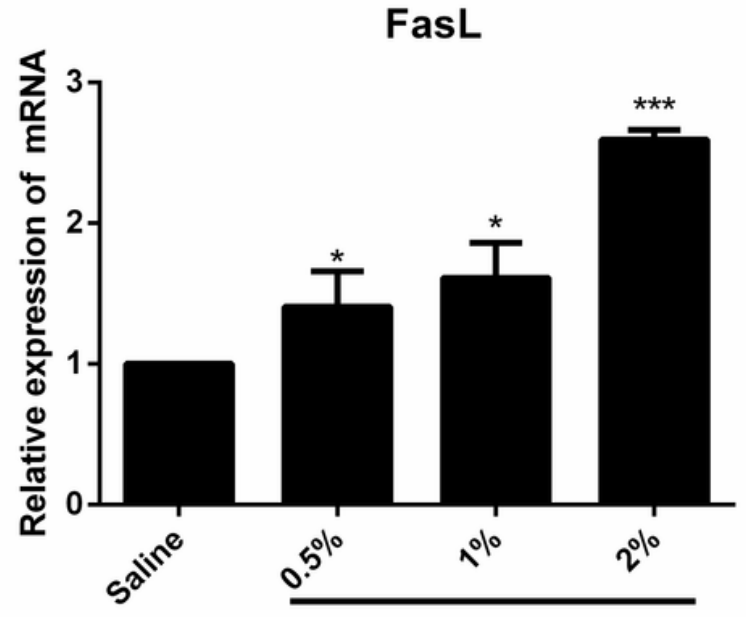

Ropivacaine concentration

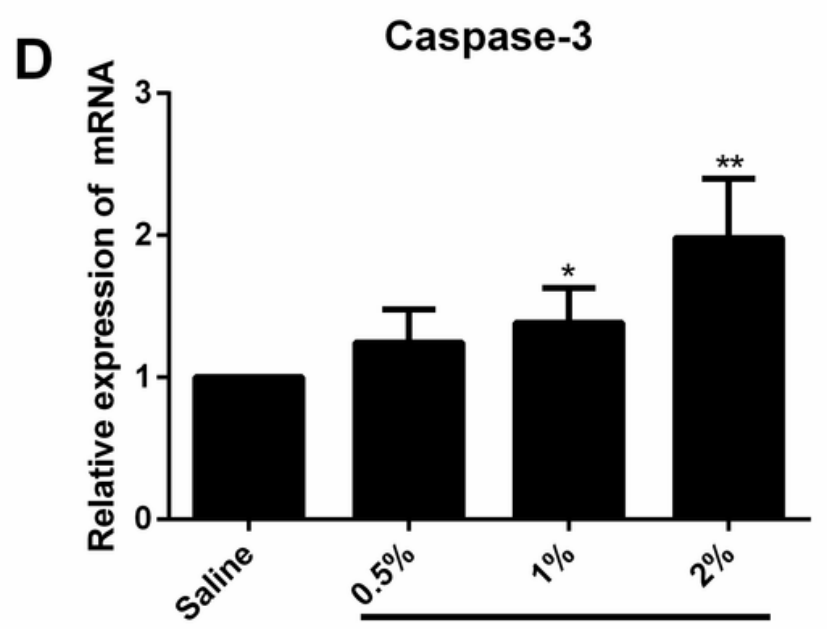

Ropivacaine concentration

Figure 5

mRNA expression of Fas, FasL, caspase-3, and caspase-8 in the spinal cord (quality real-time PCR) (mean \pm SD). A. Fas mRNA expression. B. FasL mRNA expression. C. Caspase-3 mRNA expression. D. Caspase-8 mRNA expression. ${ }^{*} P<0.05,{ }^{* * P}<0.01,{ }^{* * *} P<0.001$ vs saline group. 


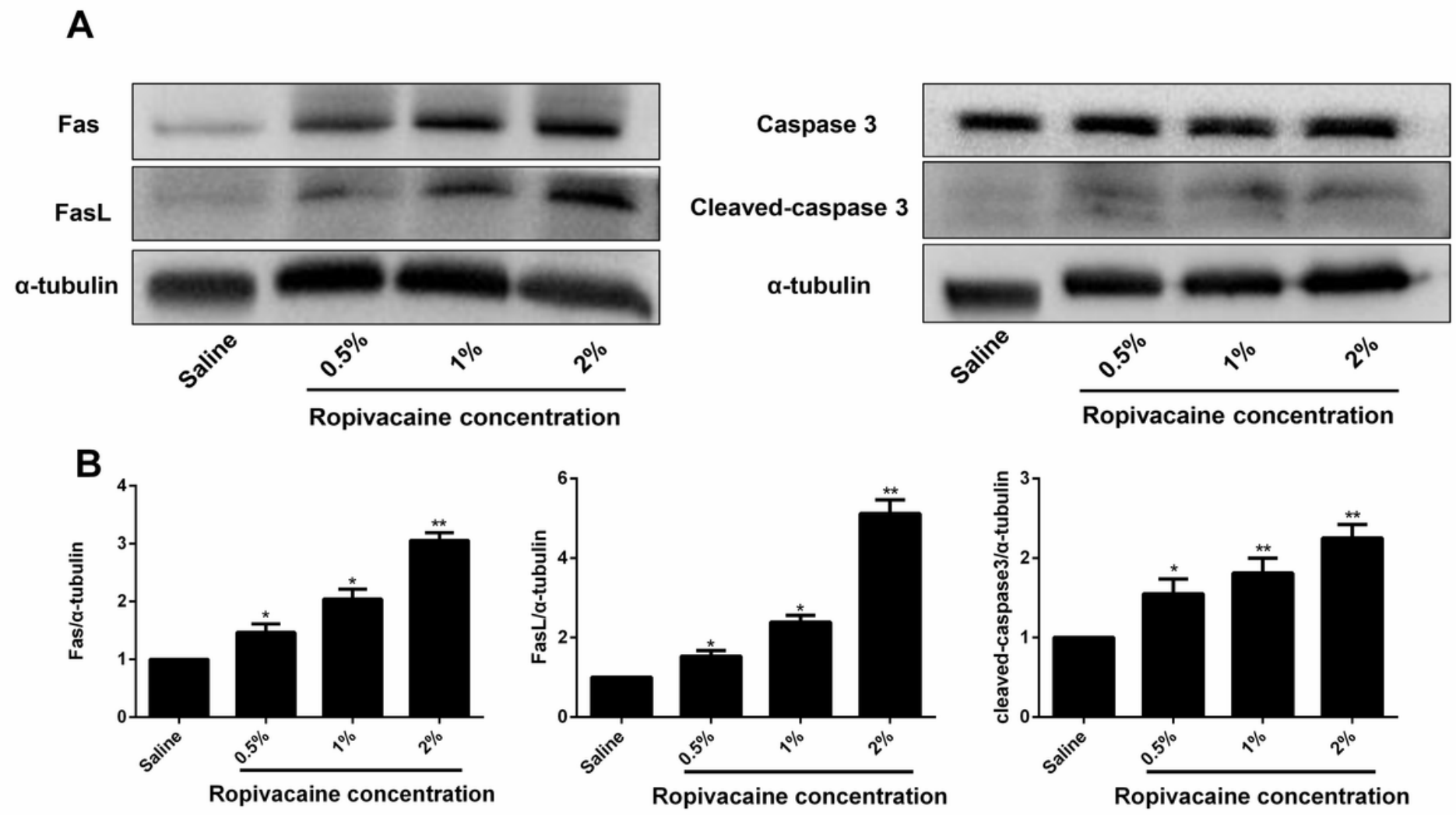

\section{Figure 6}

Protein expression of Fas, FasL, caspase-3, and cleaved-caspase-3 in the spinal cord (Western blotting) (mean \pm SD). A. Protein band of Fas, FasL, cleaved caspase-3 and a-tubulin. B. Quantification of Fas, FasL, cleaved caspase-3 and a-tubulin protein expression. ${ }^{*} P<0.05,{ }^{*} P<0.01$ vs Saline group.

\section{Supplementary Files}

This is a list of supplementary files associated with this preprint. Click to download.

- renameddd339.tif

- fasl.tif

- fas.tif

- cleavedcaspase3.tif

- cas3.tif

- atub.tif 